\title{
GRACEFUL NUMBERS
}

\section{KIRAN R. BHUTANI and ALEXANDER B. LEVIN}

\author{
Received 14 May 2001
}

\begin{abstract}
We construct a labeled graph $D(n)$ that reflects the structure of divisors of a given natural number $n$. We define the concept of graceful numbers in terms of this associated graph and find the general form of such a number. As a consequence, we determine which graceful numbers are perfect.
\end{abstract}

2000 Mathematics Subject Classification: 11B75, 05 C78.

1. Introduction. In [2], Gallian presented a detailed survey of various types of graph labeling, the two best known being graceful and harmonious. Recall that a graph $G$ with $q$ edges is called graceful if one can label its vertices with distinct numbers from the set $\{0,1, \ldots, q\}$ and mark the edges with differences of the labels of the end vertices in such a way that the resulting edge labels are distinct. A number of interesting results on graceful and graceful-like labelings are obtained in [1, 3, 4] and some other works. In this note, we give a description of natural numbers whose associated graph of divisors satisfies certain graceful-like conditions. For any natural number $n$, we construct a labeled graph $D(n)$ that reflects the structure of divisors of $n$. We define the concept of graceful number in terms of this associated graph and find the general form of such a number. As a consequence, we determine which graceful numbers are perfect.

2. Main results. Given a natural number $n$ one can generate a graph $D(n)$ that reflects the structure of divisors of $n$ as follows. The vertices of the graph represent all the divisors of the number $n$, each vertex is labeled by a certain divisor. (In what follows, we refer to the vertex of the graph $D(n)$ with label $k$ as the "vertex $k$.") If $r$ and $s$ are two divisors of $n$ and $r>s$, then there is an edge between the vertices $s$ and $r$ if and only if $s$ divides $r$ and the ratio $r / s$ is a prime number. As in the theory of graceful graphs, we label such an edge by the difference $r-s$ of the labels of its vertices. In what follows, the sum of the labels of all edges of the graph $D(n)$ is denoted by $\overline{S D}(n)$ while $S D(n)$ denotes the sum of labels of all edges of $D(n)$ except the edges terminating at $n$. (Clearly, if $n=p_{1}^{r_{1}} p_{2}^{r_{2}} \cdots p_{k}^{r_{k}}$ is the prime factorization of a natural number $n$, then $S D(n)=\overline{S D}(n)-\sum_{i=1}^{k}\left(n-n / p_{i}\right)$.)

EXAMPLE 2.1. It is easy to see that if $n=p^{r}$, where $p$ is a prime number and $r$ is any positive integer, then $\overline{S D}(n)=\sum_{i=1}^{r}\left(p^{i}-p^{i-1}\right)=p^{r}-1$ and $S D(n)=\sum_{i=1}^{r-1}\left(p^{i}-\right.$ $\left.p^{i-1}\right)=p^{r-1}-1$, so that $S D(n)<n$. The graph $D(n)$ is shown in Figure 2.1. 


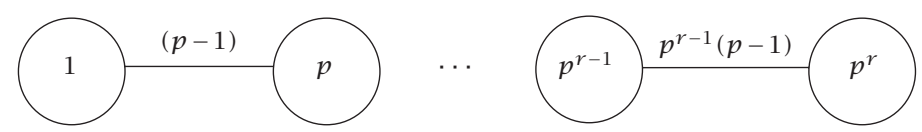

FIGURE 2.1. The graph $D\left(p^{r}\right)$.

The following example shows that there are numbers $n$ such that $S D(n)>n$, as well as numbers that satisfy the condition $S D(n)=n$.

EXAMPLE 2.2. Let $n=24$ and $m=12$. Then $S D(n)=(12-6)+(12-4)+(8-4)+$ $(6-3)+(6-2)+(4-2)+(3-1)+(2-1)=30>n$ and $S D(m)=(6-3)+(6-2)+$ $(4-2)+(3-1)+(2-1)=12=m$.

DEFINITION 2.3. A natural number $n$ is called graceful if $S D(n)=n$.

In order to obtain the description of graceful numbers, we first find the value of $S D(n)$ when $n$ is a product of powers of two different prime numbers.

EXAMPLE 2.4. Let $n=p^{r} q^{s}$ where $p$ and $q$ are different prime numbers, $r \geq 1$, and $s \geq 1$. In this case the graph $D(n)$ is of the form

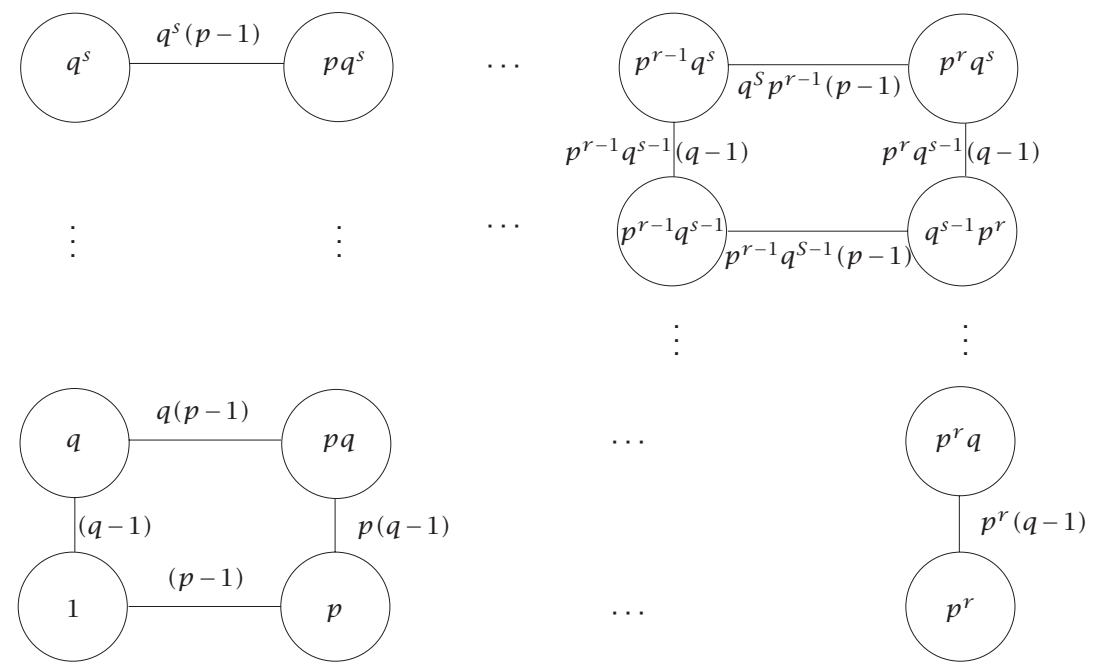

Figure 2.2. The graph $D\left(p^{r} q^{s}\right)$.

and $\overline{S D}(n)=\sum_{i=0}^{r} \sum_{j=1}^{s}\left(p^{i} q^{j}-p^{i} q^{j-1}\right)+\sum_{i=1}^{r} \sum_{j=0}^{s}\left(p^{i} q^{j}-p^{i-1} q^{j}\right)=\sum_{i=0}^{r} p^{i}\left(q^{s}-1\right)+$ $\sum_{j=0}^{s} q^{j}\left(p^{r}-1\right)$ (the first sum corresponds to the differences of the consecutive divisors of $n$ when the exponent of $q$ decreases, and the second sum takes care about the differences of consecutive divisors of $n$ when the exponent of $p$ decreases). Thus,

$$
\overline{S D}(n)=\left(q^{s}-1\right) \sum_{i=0}^{r} p^{i}+\left(p^{r}-1\right) \sum_{j=0}^{s} q^{j}=\left(q^{s}-1\right) \frac{p^{r+1}-1}{p-1}+\left(p^{r}-1\right) \frac{q^{s+1}-1}{q-1},
$$


so that

$$
S D(n)=\overline{S D}(n)-\left[\left(n-\frac{n}{p}\right)+\left(n-\frac{n}{q}\right)\right] .
$$

It follows from formulas (2.1) and (2.2) that a number $n=p^{r} q^{s}$ ( $p$ and $q$ are prime, $r \geq 1$, and $s \geq 1$ ) is graceful if and only if $p=2$ and $s=1$, that is, $n=4 q$ for some odd prime number $q$.

Indeed, equality $S D(n)=n$ can hold only for even numbers $n$ (if $n$ is odd, then (2.1) shows that $S D(n)$ is even, whence $S D(n) \neq n$ ). If $n=2^{r} q^{s}$, where $r \geq 2, s \geq 2$, then

$$
\begin{aligned}
S D(n)-n & =\left(2^{r}-1\right) \sum_{i=0}^{s} q^{i}+\left(q^{s}-1\right)\left(2^{r+1}-1\right)-2^{r+1} q^{s}+2^{r-1} q^{s}+2^{r} q^{s-1}-2^{r} q^{s} \\
& >\left(2^{r-1}-2\right) q^{s}+\left(2^{r+1} q^{s-1}-q^{s-1}-2^{r+1}\right)+\left(2^{r}-1\right) \sum_{i=0}^{s-2} q^{i} \\
& >0
\end{aligned}
$$

so that $S D(n)>n$. Finally, if $n=2^{r} q(r \geq 1)$, then $S D(n)-n=(q-1)\left(2^{r+1}-1\right)+$ $\left(2^{r}-1\right)(q+1)-2^{r+1} q+2^{r-1} q+2^{r}-2^{r} q=q\left(2^{r-1}-2\right)$, so that $S D\left(2^{r} q\right)=2^{r} q$ if and only if $r=2$. Thus, for any two different prime numbers $p$ and $q, p<q$, and for any two nonnegative integers $r$ and $s$, the number $p^{r} q^{s}$ is graceful if and only if $p=2$, $r=2$, and $s=1$.

Now, we generalize formula (2.1) to the case of arbitrary number $n$. More precisely, we show that if $n=p_{1}^{r_{1}} p_{2}^{r_{2}} \cdots p_{k}^{r_{k}}$ is a prime decomposition of a positive integer $n$ $\left(p_{1}, \ldots, p_{k}\right.$ are different primes and $r_{1}, \ldots, r_{k}$ are positive integers), then

$$
\overline{S D}(n)=\sum_{i=1}^{k}\left(p_{i}^{r_{i}}-1\right) \prod_{1 \leq j \leq k, j \neq i}\left(\frac{p_{j}^{r_{j}+1}-1}{p_{j}-1}\right) .
$$

We proceed by induction on $n$. We have seen that the formula is true if $n$ is a power of a prime number or a product of two powers of primes. In order to perform the step of induction, notice that

$$
\overline{S D}(n)=\overline{S D}\left(\frac{n}{p_{1}}\right)+\left(p^{r_{1}}-p^{r_{1}-1}\right) \sum_{i_{2}=0}^{r_{2}} \cdots \sum_{i_{k}=0}^{r_{k}} p_{2}^{i_{2}} \cdots p_{k}^{i_{k}}+p_{1}^{r_{1}} \overline{S D}\left(\frac{n}{p_{1}^{r_{1}}}\right) .
$$

Applying the inductive hypothesis and taking into account that

$$
\overline{S D}(n)=\sum_{i_{2}=0}^{r_{2}} \cdots \sum_{i_{k}=0}^{r_{k}} p_{2}^{r_{2}} \cdots p_{k}^{r_{k}}=\prod_{j=2}^{k} \sum_{i=0}^{r_{j}} p_{j}^{i}=\prod_{j=2}^{k}\left(\frac{\left(p_{j}^{r_{j}+1}-1\right)}{\left(p_{j}-1\right)}\right),
$$

we obtain that

$$
\begin{aligned}
\overline{S D}(n)= & \left(p_{1}^{r_{1}-1}-1\right) \prod_{j=2}^{k}\left(\frac{\left(p_{j}^{r_{j}+1}-1\right)}{\left(p_{j}-1\right)}\right)+\sum_{i=2}^{k}\left(p_{i}^{r_{i}}-1\right)\left(\frac{\left(p_{1}^{r_{1}}-1\right)}{\left(p_{1}-1\right)}\right) \prod_{2 \leq j \leq k, j \neq i}\left(\frac{\left(p_{j}^{r_{j}+1}-1\right)}{\left(p_{j}-1\right)}\right) \\
& +\left(p_{1}^{r_{1}}-p_{1}^{r_{1}-1}\right) \prod_{j=2}^{k}\left(\frac{\left(p_{j}^{r_{j}+1}-1\right)}{\left(p_{j}-1\right)}\right)+p_{1}^{r_{1}} \sum_{i=2}^{k}\left(p_{i}^{r_{i}}-1\right) \prod_{2 \leq j \leq k, j \neq i}\left(\frac{\left(p_{j}^{r_{j}+1}-1\right)}{\left(p_{j}-1\right)}\right)
\end{aligned}
$$




$$
\begin{aligned}
& =\left(p_{1}^{r_{1}-1}\right) \prod_{j=2}^{k}\left(\frac{\left(p_{j}^{r_{j}+1}-1\right)}{\left(p_{j}-1\right)}\right)+\sum_{i=2}^{k}\left(p_{i}^{r_{i}}-1\right) \prod_{1 \leq j \leq k, j \neq i}\left(\frac{\left(p_{j}^{r_{j}+1}-1\right)}{\left(p_{j}-1\right)}\right) \\
& =\sum_{i=1}^{k}\left(p_{i}^{r_{i}}-1\right) \prod_{1 \leq j \leq k, j \neq i}\left(\frac{\left(p_{j}^{r_{j}+1}-1\right)}{\left(p_{j}-1\right)}\right),
\end{aligned}
$$

so formula (2.4) is proved.

Now, formulas (2.2) and (2.4) imply that

$$
S D(n)=\sum_{i=1}^{k}\left(p_{i}^{r_{i}}-1\right) \prod_{1 \leq j \leq k, j \neq i}\left(\frac{p_{j}^{r_{j}+1}-1}{p_{j}-1}\right)-\sum_{i=1}^{k}\left(n-\frac{n}{p_{i}}\right) .
$$

Formula (2.8) shows, in particular, that if a number $n$ is odd, then $S D(n)$ is even (it is easily seen that both sums in the right side of the formula are even if $n$ is odd). Therefore, every graceful number must be even, that is,

$$
n=2^{r} q_{1}^{s_{1}} \cdots q_{m}^{s_{m}}
$$

for some odd primes $q_{1}, \ldots, q_{m}\left(m \geq 1, s_{i} \geq 1\right.$ for $\left.i=1, \ldots, m\right)$. As we have seen, if $m=1$, then the number $n$ is graceful if and only if $s_{1}=1$ and $r=2$, that is, $n=4 q_{1}$. We show that if $m \geq 2$, then $S D(n)>n$, so the only graceful numbers are the numbers of the form $4 q$ where $q$ is an odd prime.

First of all, notice that $S D\left(2^{r} q_{1}^{s_{1}}\right) \geq 2^{r} q_{1}^{s_{1}}$ for $r \geq 1, s \geq 2$ (see Example 2.4) and $S D\left(2 q_{1} q_{2}\right) \geq 2 q_{1} q_{2}$ for any two different primes $q_{1}$ and $q_{2}$ (applying formula (2.1) we obtain that $S D\left(2 q_{1} q_{2}\right)=\left(q_{1}+1\right)\left(q_{2}+1\right)+3\left(q_{1}-1\right)\left(q_{2}+1\right)+3\left(q_{2}-1\right)\left(q_{1}+1\right)-$ $\left.6 q_{1} q_{2}+q_{1} q_{2}+2 q_{1}+2 q_{2}=2 q_{1} q_{2}+3\left(q_{1}+q_{2}\right)-5>2 q_{1} q_{2}\right)$. Therefore, in order to prove that $S D(n)>n$ for any number $n$ of the form (2.9) with $m \geq 2$, it is sufficient to prove that $S D(n)>q_{m}^{s_{m}} S D\left(n / q_{m}^{s_{m}}\right)$. But the last inequality is a consequence of equality (2.5). Indeed,

$$
\begin{aligned}
S D(n)= & \overline{S D}(n)-n=\overline{S D}\left(\frac{n}{q_{m}}\right)+q^{s_{m}}-q^{s_{m}-1} \sum_{i=0}^{r} \sum_{i_{1}=0}^{s_{1}} \cdots \sum_{i_{m-1}=0}^{s_{m-1}} 2^{i} q_{1}^{i_{1}} \cdots q_{m-1}^{i_{m-1}} \\
& +q_{m}^{s_{m}} \overline{S D}\left(\frac{n}{q_{m}^{s_{m}}}\right)-n>q_{m}^{s_{m}}\left(\overline{S D}\left(\frac{n}{q_{m}}\right)-\frac{n}{q_{m}}\right)=q_{m}^{s_{m}} S D\left(\frac{n}{q_{m}^{s_{m}}}\right) .
\end{aligned}
$$

We arrive at the following result.

THEOREM 2.5. A natural number $n$ is graceful if and only if $n=4 q$ where $q$ is an odd prime.

Recall that a positive integer $m$ is called a perfect number if it is equal to the sum of all its proper divisors (i.e., of all divisors of $m$ except of the number $m$ itself). It is known (cf. [4, Theorem 5.10]) that every even perfect number is of the form $2^{k-1}\left(2^{k}-\right.$ 1 ), where the number $2^{k}-1$ is prime. Thus, our theorem implies the following result.

COROLLARY 2.6. The only perfect graceful number is 28. 


\section{REFERENCES}

[1] R. W. Frucht, Nearly graceful labelings of graphs, Sci. Ser. A Math. Sci. (N.S.) 5 (1992/93), 47-59.

[2] J. A. Gallian, A dynamic survey of graph labeling, Electron. J. Combin. 5 (1998), no. 1, Dynamic Survey 6, 1-43.

[3] D. Moulton, Graceful labelings of triangular snakes, Ars Combin. 28 (1989), 3-13.

[4] A. Rosa, On certain valuations of the vertices of a graph, Theory of Graphs (Internat. Sympos., Rome, 1966), Gordon and Breach, New York, 1967, pp. 349-355.

KirAn R. BHUtANi: DePARTMENT OF MATHEMATICS, THe CATHOLIC UNIVERSity OF AMERICA, WASHINGTON, DC 20064, USA

E-mail address: bhutani@cua.edu

Alexander B. LeVin: Department of Mathematics, The Catholic University of AMERICA, WASHINGTON, DC 20064, USA

E-mail address: 1evin@cua.edu 


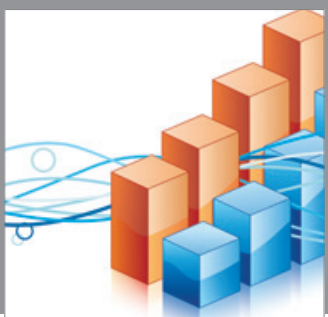

Advances in

Operations Research

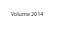

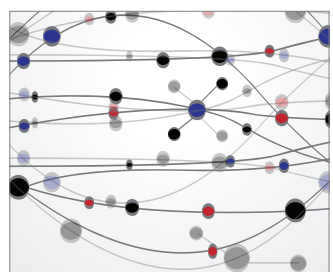

\section{The Scientific} World Journal
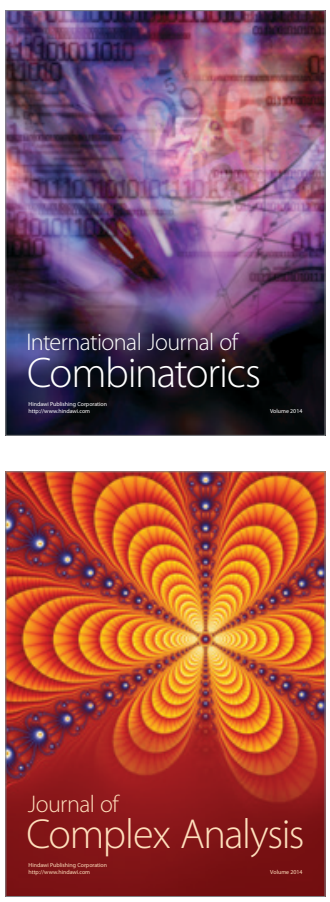

International Journal of

Mathematics and

Mathematical

Sciences
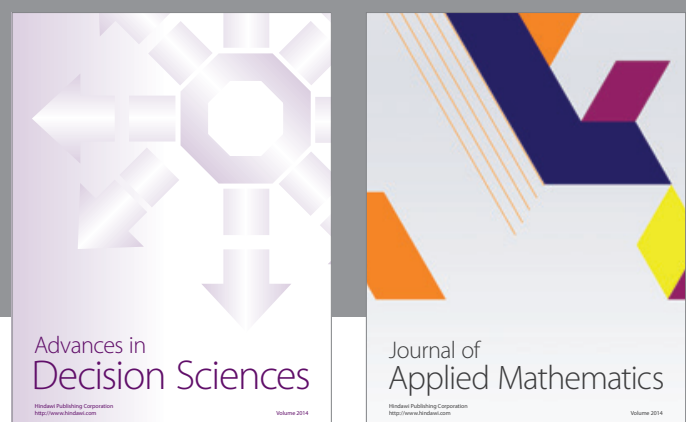

Journal of

Applied Mathematics
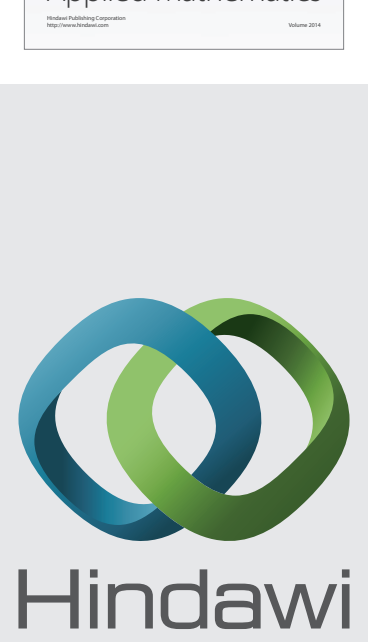

Submit your manuscripts at http://www.hindawi.com
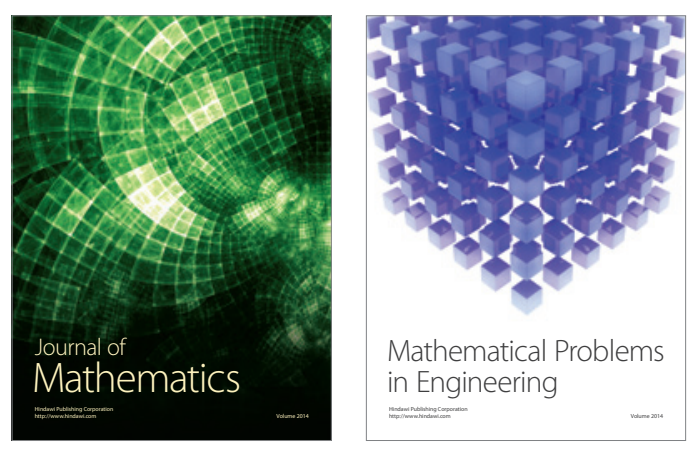

Mathematical Problems in Engineering
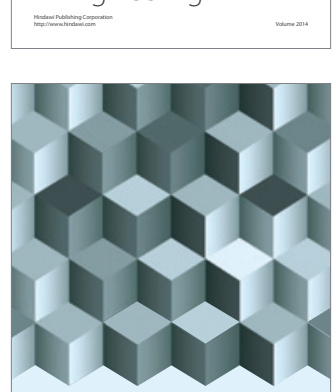

Journal of

Function Spaces
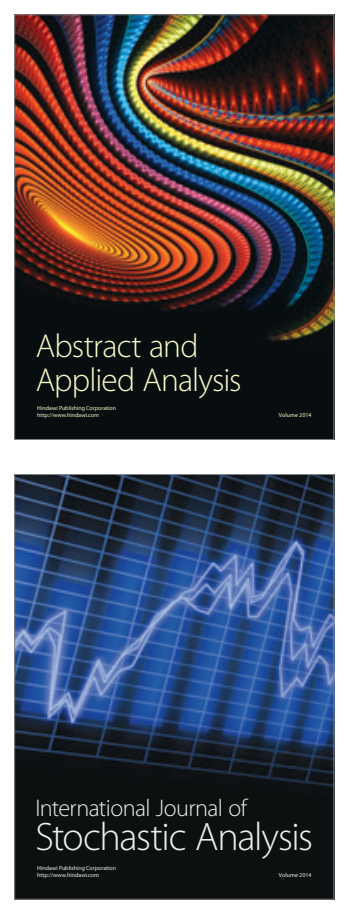

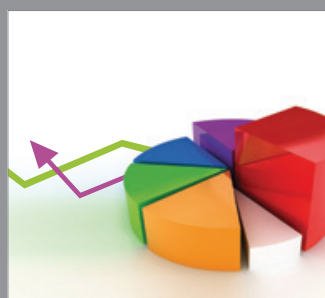

ournal of

Probability and Statistics

Promensencen
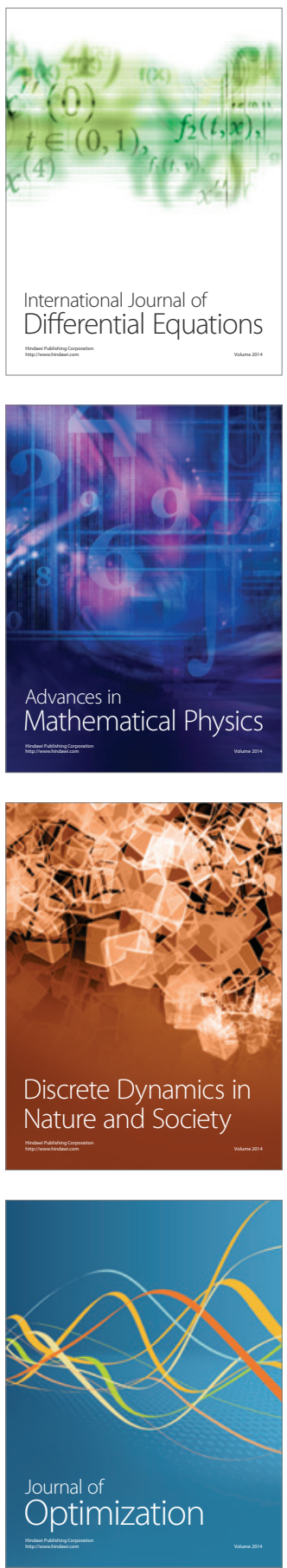